\title{
Explanatory investigation of the device for regulating the vertical position of a building
}

\author{
Aleksandr Petrakov, Ekateryna Bryzhata*, and Nikolay Maslo \\ Donbass National Academy of Civil Engineering and Architecture, Makeyevka, Ukraine, 286123
}

\begin{abstract}
The article is devoted to the study of the strain-stress distribution of the frame elements of a multi-storey building with the correction of the tilt using a device for control the vertical position. The construction of the device for adjusting the geometric position of the building, which undergoes deformation of the ground base, is considered. Quoted results of experimental researches of the developed device for correction the tilt of a building. The influence of the loading level, sand moisture, the height of the intersection of the slots and the sand composition on the efficiency of the tilt correction device is analyzed.A finite-element model of a multi-storey building with constructive measures of protection against the influence of above permitted standard tilts, taking into account the interaction of the structure with the deformable ground base and the construction of foundations with variable dimensions are developed.The schemes for the multi - stage correction of the tilt of the finite - element model of the building are investigated.
\end{abstract}

\section{Introduction}

Experience shows that one of the main problems in the use of buildings and structures in large cities is the possibility of their damage as a result of non-uniform deformation of the ground base. Difficult engineering-geological conditions are thus the main cause of instability as a reason under construction and existing facilities, which increases the risk of loss of load capacity. In this regard, of particular importance is the problem of the technical state of the control bearing structures in order to prevent emergency situations and the validity of the choice of a complex of engineering measures for their prevention. It is obvious that control of the technical condition of load-bearing structures should be systematic and allow for the assessment of the changes taking place on the basis of quantitative criteria.

The problem of protection of buildings and structures from the harmful influence of the tilt is quite complicated, requiring a deep understanding of the causes and knowledge of the degree of responsibility for the protected object and its basic parameters. The scope protection of buildings and structures may differ materially from the tilt in a variety of conditions. In some cases, it is not feasible to perform it, in others, the cost of such activities may amount to more than ten percent of the estimated cost of the projected facility.

*Corresponding author kate88.88@bk.ru 
Consequently, the problem of protecting buildings and structures from tilt is not only technical or engineering-geological, but also economic.

Methods of calculation of buildings and structures in difficult geological conditions considered in the works of scientists [1-13]. Methods for correcting tilts of buildings by lifting their parts with hydraulic jacks are considered in the works of scientists [15-20]. Methods for correcting tilts of buildings by changing the properties of the ground bases (drilling, additional loading, etc.) are considered in the works [21-25].

The purpose of the work is the development of new construction with variable parameters (devices) for the correction of tilts of buildings and structures based on the principle of regulating the geometric position of buildings and structures. Investigation of the stress-strain state of the frame building elements on a slab foundation while adjusting the geometric position of the building. To achieve this purposes, the following research objectives are set:

1. Develop a construction of the device with a variable height for adjusting the vertical position of a multi-storey building.

2. Investigate the geometric parameters of the device for correcting the tilts of the buildings, affecting the deformability of the test medium. Carry out an experimental analysis of the device under different loading conditions and design magnitudes of its height.

3. To analyze the design models of frame buildings on an elastic foundation and to justify by numerical studies technological schemes for correcting tilts of buildings using structures with variable parameters.

\section{Methods}

Developed construction of the device for adjusting the geometrical position of the building, which undergoes ground base deformation is shown in Figure 1
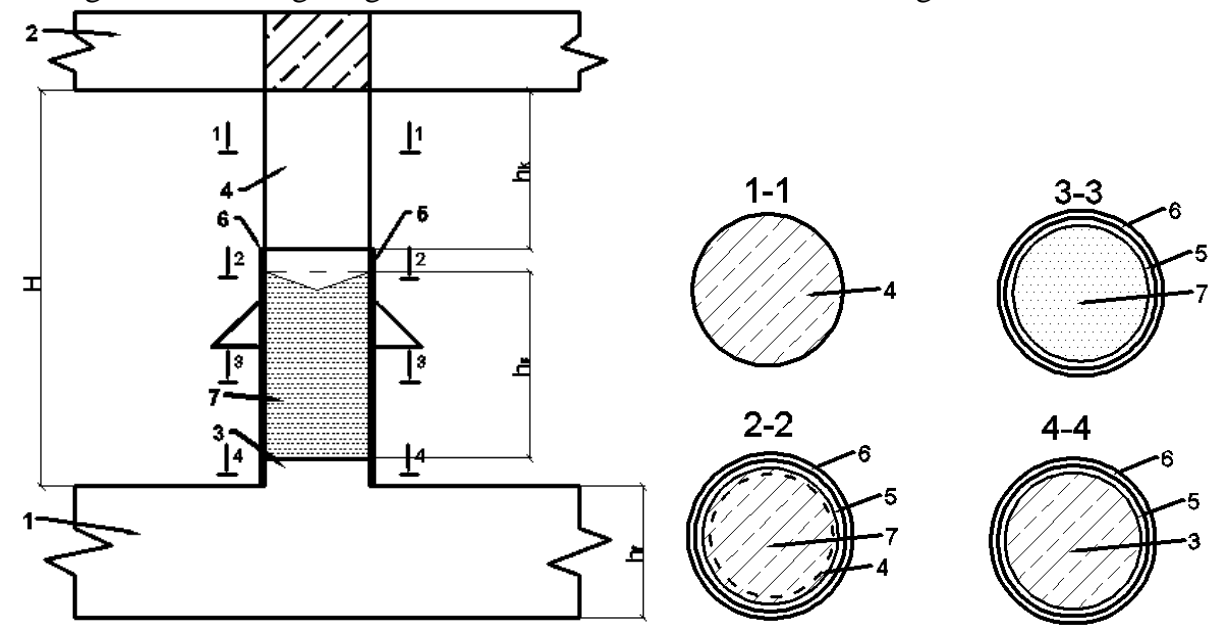

Fig. 1. Structure of the foundation with a device for correcting the tilts of structures: 1 - foundation slab; 2 - beam-and-girder construction; 3 - plain spigot from foundation slab; 4 - tubular element from shotcrete, embedded in the beam-and-girder construction; 5 - inner tube of the device; 6 - outer tube of the device; 7 - test medium (sand); $\mathrm{H}$ - height of the technical underground; $\mathrm{h}_{\mathrm{s}}-$ height of the test medium in the device; $h_{\kappa}-$ standoff between the device and the bottom of the beam-and-girder construction. 
The foundation is a solid reinforced concrete slab and a beam-and-girder construction, divided by the height of technological area to accommodate the device to adjust the geometric position of the building.

Developed device consists of two metal tubes: inner and outer, which have intersecting at right angles slots (figure 2). Between tubes applied black-lead lubrication. Inner tube of the device set on plain spigot from foundation slab. In the inner tube is fed test medium in the form of backfill sand, which is compacted by vibrostamp. The height of the test medium is equal to the maximum value of the vertical adjustment of the structure, increased by $10 \%$. In the upper part of the inner tube over the test medium set tubular element from shotcrete, which are merged into a system of crossbeams. Columns frame are mounted on the cross-beam system.

The outer tube of the device has stops (knobs) for rotational displacement of the tube. Initially, the inclined slots do not intersect in the tubes and sand is in a state of compression ratio. To carry out the vertical movement of the upper part of the foundation opens the hole in the apparatus by rotating the outer tube. The size of the hole must be at least $10 \times 10$ $\mathrm{mm}$. Lowering of the upper part of the foundation occurs at the expense of the expiration of the sand from the device.

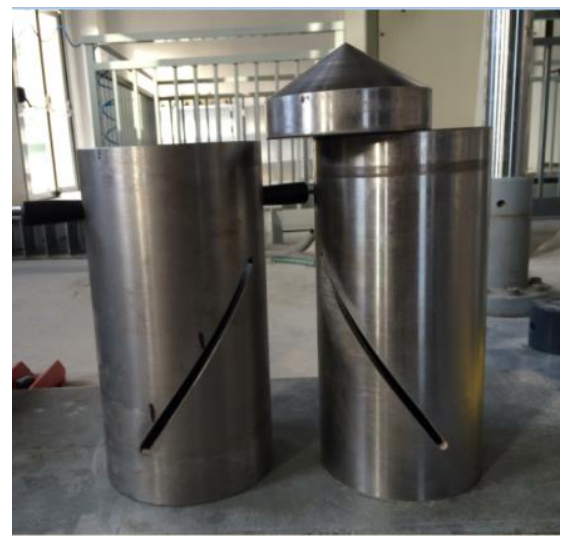

Fig. 2. Models of shells (tubes) of the device with intersecting slots on the side surfaces

The height of the tubular element, embedded in the beam-and-girder construction, should provide a gap between the bottom beams and the top of inner tube, equal to the maximum straightening value of the construction, increased by $10 \%$. Sealing elements and in the device should be not less than the internal diameter of the inner tube.

As a stamp, a tapered tip is selected, because cone is the most successful form of an axisymmetric cutting profile. The conical shape of the sole creates a wedging effect in the granular material, which outflow from the hole (figure 3). The tapered tip allows the creation of lateral pressures that sufficient to expel sand from the hole. The height of the cone should not be less than the calculated outflanking of the device at the stage, which in experiments is $30 \mathrm{~mm}$. 

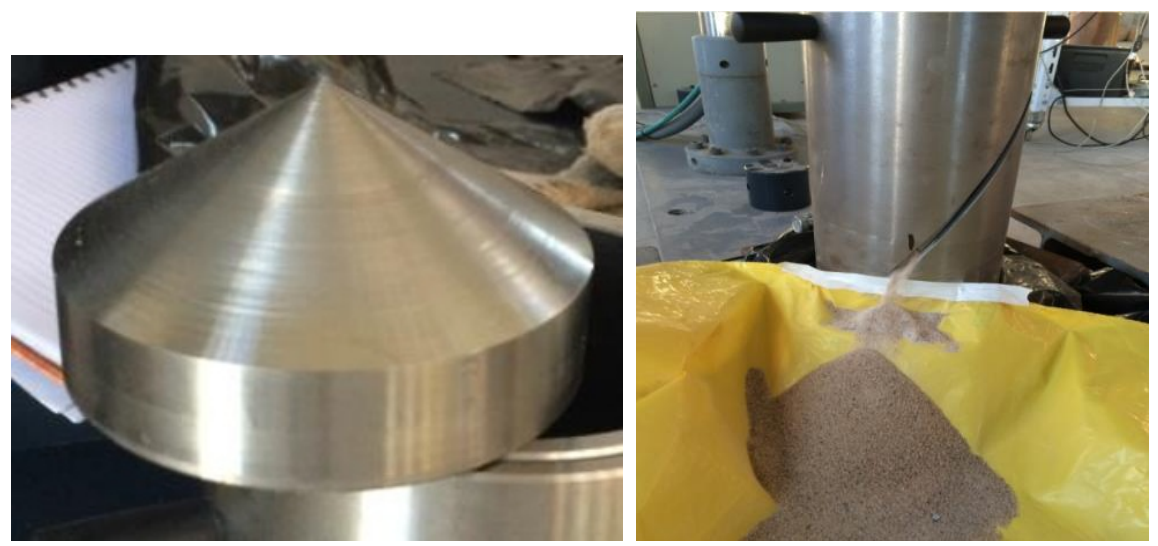

Fig. 3. Model of tapered tip of a stamp and expiration of sand from the model of the device during testing

Before beginning of the experiment, preliminary sand loading was carried out with the maximum pressure in the experiments. After that, the magnitude of the required load was set $3,7,15,40$ tons for different sand moisture $0,3,5,10$ and $15 \%$, for different height of intersections of the holes on the device 3, 6, 9, 12, 15, 18, 21, 24, 27 and $30 \mathrm{~cm}$.

Methods of theoretical research suggest the study of a 14-story frame building on a slab foundation (figure 4). The methods are based on the use of design models that take into account the interaction of structures with a deformable ground base and the construction of foundations with variable in the process of calculating the dimensions. Constructions of foundations with variable in the process of calculating the dimensions are used to simulate the process of levelling the building by lowering its less sagging parts. In these studies, the design of a foundation with variable dimensions is a device for regulating the tilts of a structure. Its height decreases during the expiration of the sand from the device. In the analytical model of the device for the correction of tilts, structures are modelled combined finite elements with a given axial rigidity. The decrease in the height of the device in the calculations is taken into account as the temperature deformation specified in the statically determinate principal system.

The main differences in the methods of calculating the system «ground base foundation- superficial structure» for the correcting the spatial position of the structure are refined definitions of the calculated combinations of loads and impacts. In this case, the main, special and technological combinations of loads and impacts are considered.

The main combination are taken into account the operational loads applied to the structures at the time before the correction of the spatial position of the building begins.

The special combinations of loads include the load of the main combination and the uneven sediments of the ground base, which were manifested at the time of the beginning of the work for levelling the building.

The technological combinations of loads include main and special combinations and one of the technological impacts in the form of forced displacements in bar that simulate the device. The number of technological combinations of loads corresponds to the number of stages for levelling the building. 


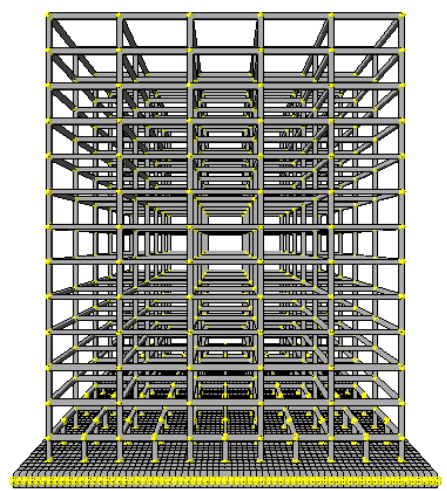

Fig. 4. Analytical model of the 14-story frame building on a slab foundation (PC LIRA)

The technological schemes for correcting the spatial position of the 14-storey building by multi-stage lowering of its less sagging parts by $3-6 \mathrm{~cm}$, and by multi-stage sequential lowering of its parts along the axes of the columns in the full-size non-uniform precipitation at $30 \mathrm{~cm}$.

\section{Results and discussions}

Experimental studies were carried out in order to determine the influence of the loading level, sand moisture and composition of sand for efficiency of the proposed device [26-30]: effect of preloading (figure 5), sand moisture (figure 6), the loading time and the state of the sand for deformability (figures 7,8).

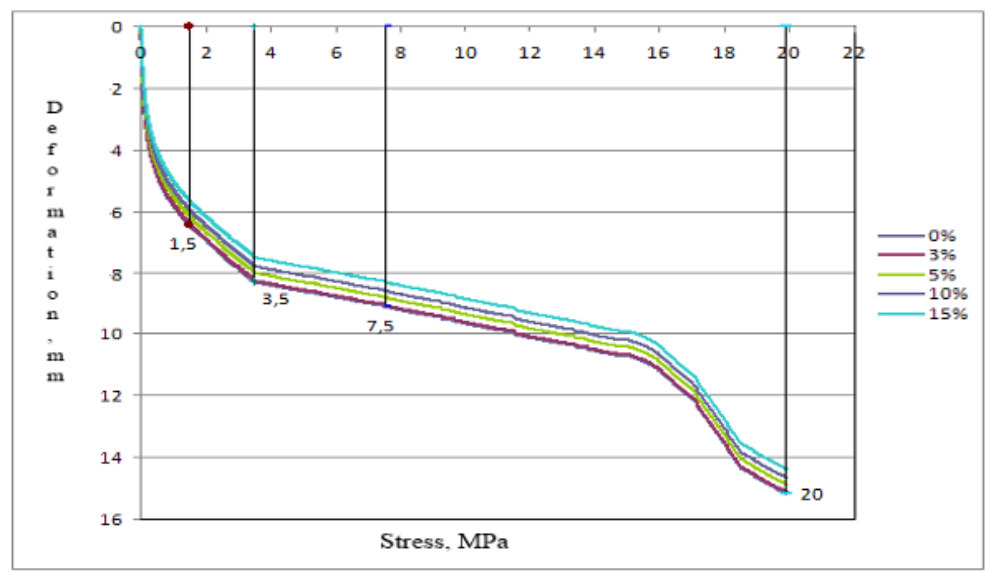

Fig. 5. Effect of preload on the deformability of sand at different sand moisture 


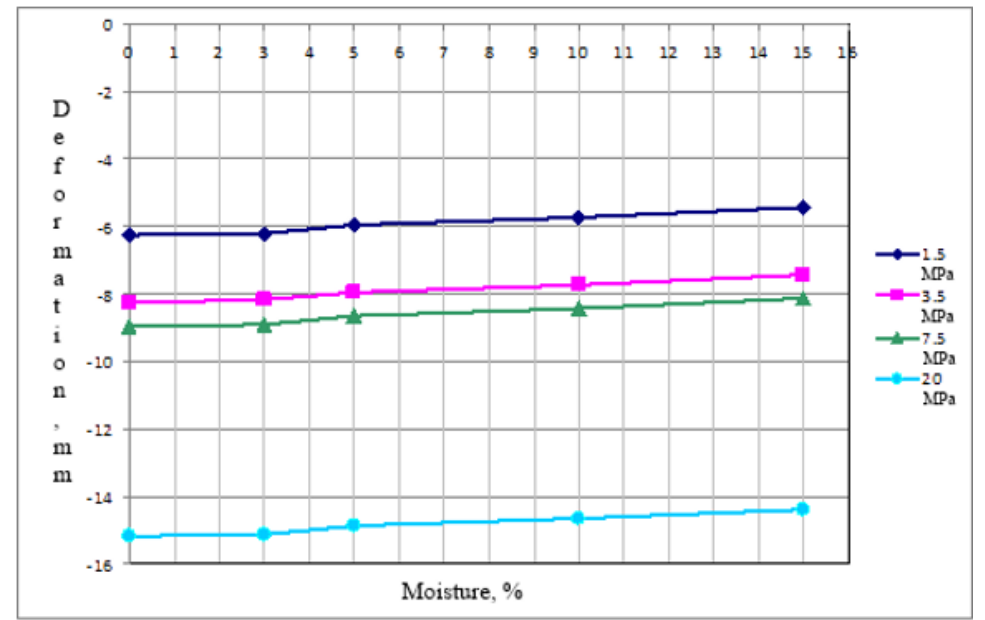

Fig. 6. The influence of sand moisture on the deformability of sand at different stresses

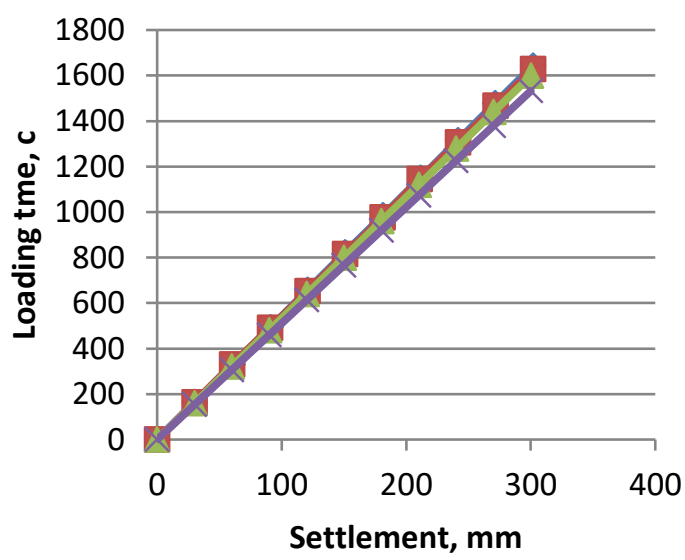

Fig. 7. Sand expiration time to achieve desired settlement

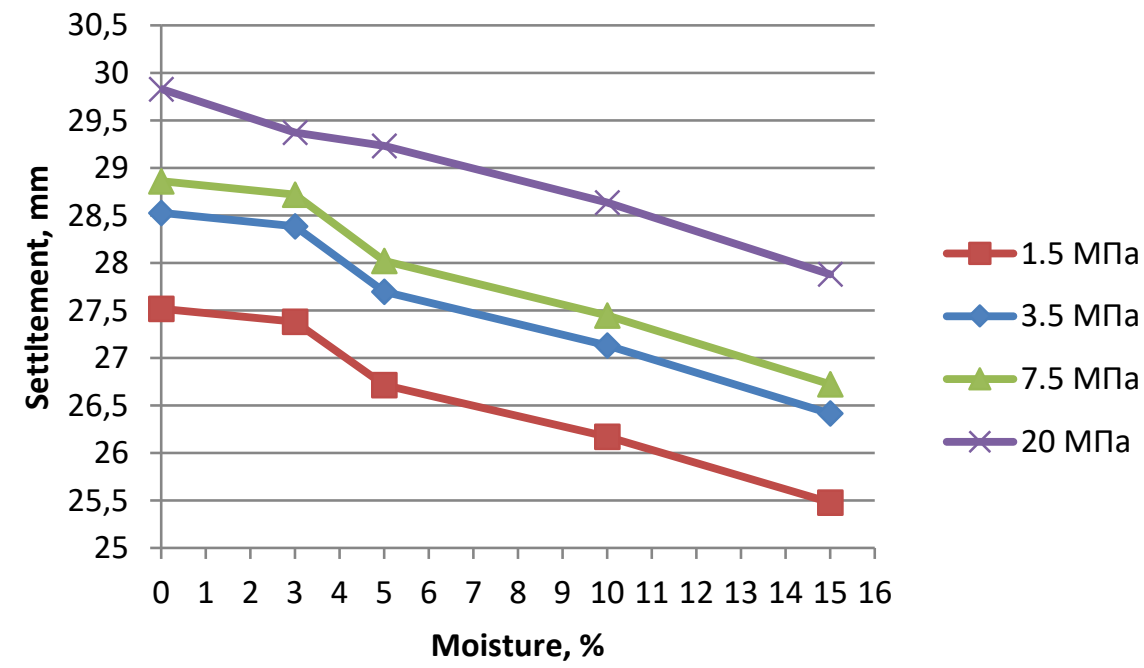

Fig. 8. Influence of the sand moisture level on the settlement of the device (operation time $150 \mathrm{~s}$ ) 


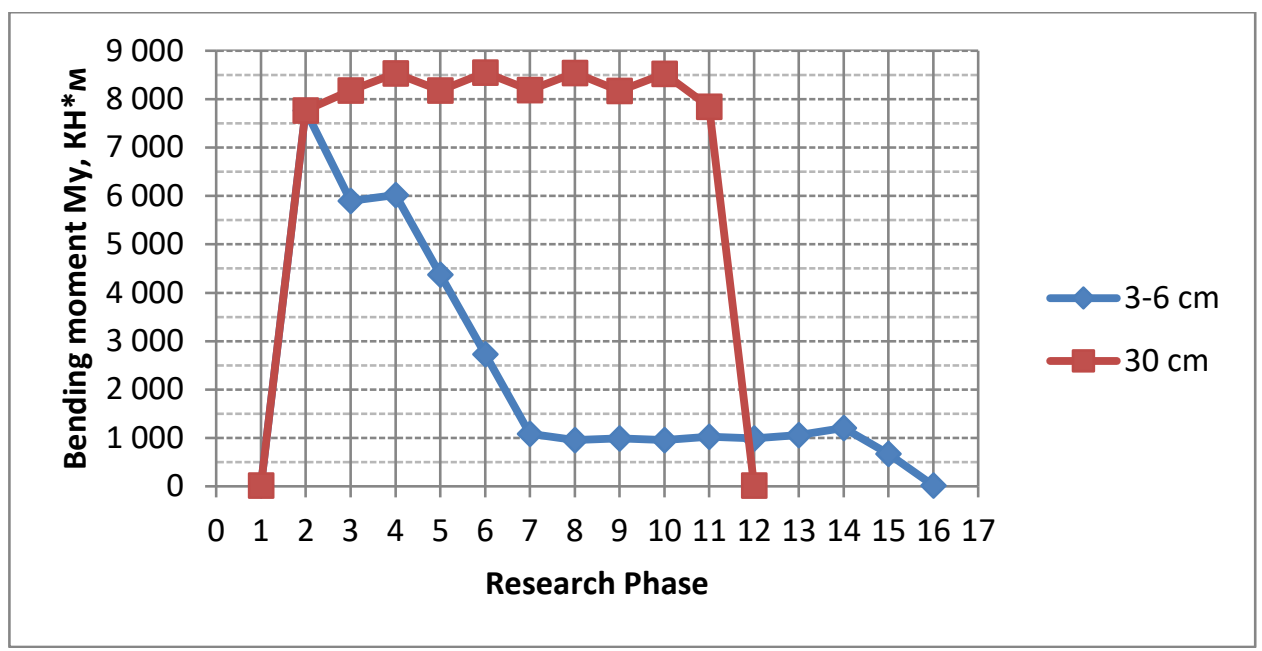

Fig. 9. The maximum value of the bending moment in the studied elements of the frame building with a multistage alignment of $3-6 \mathrm{~cm}$ and $30 \mathrm{~cm}$

It has been established that with a multistage alignment of 3-6 cm, a reduction in effort is observed at each stage of the study by $30-60 \%$. Thus, the operation of the device when the precipitate for 3-6 $\mathrm{cm}$ for each phase equalization leads to gradual reduction in the effort. With the gradual alignment of the column axes by $30 \mathrm{~cm}$, significant additional efforts are made in the elements of the building structure, which can lead to the destruction of individual structures or the entire building. At the same time, in the process of levelling the building, the efforts in the constructions not only do not decrease, but rather increase by $5-7 \%$. The strain-stress state of building constructions is stabilized only at the last stage of levelling, when the foundations of all columns are located on the same level.

\section{Conclusions}

1. Device for regulating the vertical position of a building with uneven deformations of the ground base by the method of lowering its parts has been developed and investigated.

Experimental researches established that the device's working capacity is ensured by observing the following technological principles:

- the pressure on the test medium must be created by means of a stamp with a conical base, creating lateral pressures in the sand, sufficient for its outflow from the hole in the device;

- the hole for the outflow of sand during the operation of the device must be forced to move discretely along the height of the device to the height of the conical die base.

Failure to comply with these principles leads to wedging of the test medium in the volume of the device.

2. The results of the experiments showed a slight dependence (less than 10\%) the values of the stress in the sand on the values of the draft of the stamp. This is because the outflow of sand from the device occurs when the hole is located in the zone of the conical stamp, in which the maximum lateral pressures are created from the vertical load applied to the die. Expiration of sand from the hole begins when the lateral pressures reach a certain value (the threshold of expiration). In experiments this value is $1.5 \mathrm{MPa}$. It can be assumed that, at elevated lateral pressures, there is partial seizure of the sand in the hole. Thus, at lateral pressures from different vertical loads on the die in experiments, the rate of outflow of sand from the hole was approximately the same. 
In the conducted experiments, the sludge of the stamp in the device by $30 \mathrm{~mm}$ was reached in $160 \mathrm{~s}$ of its operation. Complete precipitate die $300 \mathrm{~mm}$ achieved for the operation of the device $1600 \mathrm{~s}$. Thus, in preliminary calculations, the average deposition rate of the die in the device can be assumed to be $0.2 \mathrm{~mm} / \mathrm{s}$.

3. The technological schemes of fixing the building tilts by the method of lowering its parts are investigated.

Numerical studies have substantiated the technical and technological feasibility of a step-by-step process of fixing the tilt of a building with steps of one-stage shortening of devices by $3-6 \mathrm{~cm}$. This technological scheme of fixing the building tilt completely corresponds with the technical parameters of the device for correcting the tilt of the building, which allows in automatic mode to discretely reduce the height of supports (devices) by 3-6 cm.

The data obtained from the results of theoretical and experimental studies of the efficiency of technological processes using controlled foundations, allowed to introduce a number of refinements and additions to existing methods of numerical modelling of buildings on regulated foundations. These refinements relate to finite-element models and numerical research methods.

\section{References}

1. A. Shashkin, K. Shaskin, The interaction of the building and grounds, 2 Pp.7-8 (2002)

2. O. Shulyatyev, Vestnik PNINU, 4 Pp. 203-245 (2014)

3. M. Marschalko, I. Yilmaz, M. Bednárik, K. Kubečka, Doubrava Vrchovec, F. López Gayarre, M.I. Álvarez-Fernández, C. González-Nicieza, A.E. Álvarez-Vigil, G. Herrera García, Engineering Failure Analysis, 17 Pp. 270-285 (2010)

4. A.E. Álvarez-Vigil, C. González-Nicieza, F. López Gayarre, M.I. ÁlvarezFernández, Engineering Failure Analysis, 17 Pp. 938-960 (2010)

5. X. Jie, Z. Jin, Trans. Nonferrous Met. Soc. China, 21 Pp. 516-522 (2011)

6. M.I. Álvarez-Fernández, A.E. Álvarez-Vigil, C. González-Nicieza, F. López Gayarre, Engineering Failure Analysis, 18 Pp. 1295-1307 (2011)

7. A. Yerro, J. Corominas, D. Monells, J. J. Mallorquí., Engineering Geology, 170 Pp. 52-65 (2014)

8. C. Wu, H. Hao, Soil Dynamics and Earthquake Engineering, 25 Pp. $27-38$ (2005)

9. G. Ma, H. Hao, Y. Zhou. Engineering Structures, 22 Pp. 1378-1389 (2000)

10. H.J. Ma., S.T. Quek, K.K. Ang, Engineering Structures, 26 Pp. 1661-1675 (2004)

11. M. Son, Tunnelling and Underground Space Technology, 48 Pp. 156-169 (2015)

12. L. E. Vallejo, M. Shettima, Engineering Geology, 43 Pp. 119-133 (1996)

13. Yu. Kalugin, D. Keck, J. Pronozin, Mag. Civ. Eng., 7 (75) Pp. 139-149 (2017)

14. E. Sorochan, M. Zotov, Soil Mechanics and Foundation Engineering, 3 (41) (2004)

15. V. Zotov, L. Panasyuk, Yu. Bolotov, M. Zotov, E. Sorochan, Soil Mechanics and Foundation Engineering, 5 (39) (2002)

16. V. Zotov, M. Zotov, Reconstruction of cities and geotechnical construction, $9 \mathrm{Pp}$. 156-167 (2005)

17. V. Zotov, L. Panasyuk, E. Sorochan, Yu. Bolotov, M. Zotov, Base, foundation and soil mechanics, 5 Pp. 22-25 (2002)

18. V. Zotov, Yu. Bolotov, K. Mahviladze, M. Zotov, Georgian engineering news, 1 Pp. 111-118 (2004)

19. E. Sorochan, M. Zotov, Base, foundation and soil mechanics, 3. Pp. 14-17 (2004) 
20. V.Shumovskiy, Buildings and structures in difficult engineering and geological conditions, Pp. 91-97 (1982)

21. V. Diba, I. Krasnopolkiy, Vestnik VOLGGASU, 4 (40) Pp.1-2 (2015)

22. V. Zotov. Georgian engineering news, 1 Pp. 66-71 (2002)

23. E.Sorochan, E.Ruzhkov, Base, foundation and soil mechanics, 1 Pp. 16-18 (1979).

24. A. Popov, Mag. Civ. Eng., 4 Pp. 15-27 (2015)

25. V. Utkin, Mag. Civ. Eng., 1 Pp. 4-13 (2016)

26. A. Denisov, Mag. Civ. Eng., 73 Pp. $70-87$ (2017)

27. R. Ibragimov, S. Pimenov, Mag. Civ. Eng., 62 Pp. 3-12 (2016)

28. A. Gaifullin, R. Rakhimov, N. Rakhimova, Mag. Civ. Eng., 59 Pp. 66-73 (2015)

29. I. Susoeva, T. Vahnina, A. Titunin, J. Asatkina, Mag. Civ. Eng., 71 Pp. 39-50 (2017)

30. S. Klyuev, A. Klyuev, A. Abakarov, E. Shorstova, N. Gafarova, Mag. Civ. Eng., 75 Pp. 66-75 (2017) 